\title{
Morphological and anatomical features of narrow-leaved lavender plants with prolonged conservation under in vitro genebank
}

\author{
Valentina Brailko ${ }^{1 *}$, Natalya Ivanova ${ }^{1}$, Irina $Z_{\text {Zhdanova }}{ }^{1}$, and Olga Mitrofanova ${ }^{1}$ \\ ${ }^{1}$ Federal State Funded Institution of Science "The Labor Red Banner Order Nikita Botanical Gardens \\ - National Scientific Center of the RAS”, Plant Developmental Biology, Biotechnology and Biosafety \\ Department, 298648 Yalta, Russian Federation
}

\begin{abstract}
Optimization of plants long-term conservation under in vitro conditions and identification of their structural and functional features during preservation is the basis for the creation of a genebank of cultured and wild plant species in vitro. The aim of our work was to study morphological and anatomical features of plants in lavender cultivar 'Sineva' after two years of in vitro storage at low positive temperatures. The plant material was evaluated after 24 months of conservation. A significant decrease in the linear parameters of leaves in the preserved plants was noted. Leaf shape altered from narrowly linear to oblong. With prolonged storage, the thickness of the leaf blade slightly increased. The integumentary tissues were thinner, compared with the plants cultured under standard in vitro conditions, the cuticle was not clear. The number of stomata decreased. The number of small vascular bundles decreased. The obtained data demonstrate viability of in vitro preserved explants, despite significant differences in structure, preservation of chlorophyll-containing tissues, a decrease in transpiration, and an increase of trichomes number. The stability of the vegetative organs functioning was revealed.
\end{abstract}

\section{Introduction}

The main task in biological diversity conservation is the comprehensive studies and preservation of genetic resources by replenishing and maintaining collections of living plants, as well as long-term conservation of explants that ensure their viability and stability $[1,2]$. Scientific experience indicates that plants, in various culture conditions, can both preserve morphology and anatomy characteristics of a particular genotype, and acquire new features [3-5]. The information available in the publications does not provide a complete picture of the structural and functional changes that occur when plants are conserved in a genebank in vitro $[1,6,7]$. At the same time, there is a close correlation between these features and viability of stored explants. Optimization of the conditions for in vitro plant long-term conservation and identification of structural and functional features under their storage is the basis for the conservation of valuable genotypes and the creation of the in vitro plant genebank $[6,8,9]$.

\footnotetext{
${ }^{*}$ Corresponding author:valentina.brailko@yandex.ru
} 
In the Nikita Botanical Gardens, an in vitro collection of ornamental, aromatic and fruit plant cultivars has been created [10]. However, constant replenishment of the collection requires new approaches to in vitro preservation of the valuable genotypes, including the analysis of the adaptive capacity in the explants of particular plant species and cultivars under conditions of prolonged conservation.

The object of our work was to identify special morphological and anatomical features in Lavandula angustifolia Mill. plants (on the example of the cultivar 'Sineva') after prolonged conservation under the conditions of in vitro genebank.

\section{Materials and methods}

The studies were carried out in the Plant Biotechnology and Virology Laboratory of the Plant Developmental Biology, Biotechnology and Biosafety Department, Federal State Funded Institution of Science "The Labor Red Banner Order Nikita Botanical Gardens National Scientific Center of the RAS" (NBG-NSC). Methods developed in the Plant Biotechnology and Virology Laboratory were used in the presented work [10].

Meristems 0.5-0.7 mm length were excised from the vegetative buds under a binocular microscope Nikon SM2745T (Japan) and placed on MS culture medium [11]. Viral infection was eliminated by addition $10 \mathrm{mg} \mathrm{L}^{-1}$ virocid ribavirin (Virazole, 1- $\beta$-D-Ribofuranosyl-1,2,4triazole-3-carboxamide, Sigma, USA) to induction medium. For morphogenesis induction MS medium was supplemented with 0.3-1.5 $\mathrm{mg} \mathrm{L}^{-1}$ 6-benzylaminopurine (BAP, Sigma, USA), 0.3-1.5 mg L $\mathrm{m}^{-1}$ 6-furfurylaminopurine (kinetin, Sigma, USA), $0.025 \mathrm{mg} \mathrm{L}^{-1} \alpha$-naphthylacetic acid (NAA, Duchefa Biochemie, Holland) in addition to $0.25 \mathrm{mg} \mathrm{L}^{-1}$ gibberellic acid $\left(\mathrm{GA}_{3}\right.$, Duchefa Biochemie, Holland) [12]. All culture media were supplemented with $30 \mathrm{~g} \mathrm{~L}^{-1}$ sucrose and $9 \mathrm{~g} \mathrm{~L}^{-1}$ agar-agar (Panreac, Spain).

Microshoots cultured in vitro for 6 months were used for conservation. Under sterile conditions, $1.0 \mathrm{~cm}$ long segments without leaves were separated and placed in the chemical flasks $100-150 \mathrm{ml}$ on $1 / 4 \mathrm{MS}$ medium supplemented with growth inhibitors: $0.2 \mathrm{~g} \mathrm{~L}^{-1} \mathrm{CCC}$ chlorocholinchloride (BASF, Germany) and $60 \mathrm{~g} \mathrm{~L}^{-1}$ sucrose. Macro- and microelements, vitamins, and $9.0 \mathrm{~g} \mathrm{~L}^{-1}$ agar-agar were also added in the medium. Retardant-free $1 / 4 \mathrm{MS}$ medium supplemented with $60 \mathrm{~g} \mathrm{~L}^{-1}$ sucrose was used as the control. The flasks were maintained in refrigerators LIEBHERR FKvsl 4113 (Austria) with a low positive temperature of $6^{\circ} \mathrm{C}$, under light intensity $1.25-3.75 \mu \mathrm{mol} \mathrm{m} \mathrm{m}^{-2} \mathrm{~s}^{-1}$, and 16-hour photoperiod. The experiments were made three times in ten replications. Temporary histological slides were made according to the standard methods [13]. They were studied with CX41 light microscope (Olympus, Japan) equipped with SC 50 camera (Olympus, Germany) and Cell Sens Imaging Software version 1.17. A comparative analysis of structural changes was carried out with respect to the lavender plants cultured under standard in vitro conditions for 6 months. The measurements were made on the sections of 50 leaf blades.

Data statistic analysis was made with common methods using the standard software Microsoft Office Excel (2010).

\section{Results and discussions}

Our previous studies revealed the main parameters that affect the reduction of the growth kinetics and the preservation of the physiological stability in lavender plants under 24-month conservation: culture medium $1 / 4 \mathrm{MS}$ supplemented with $0.2 \mathrm{mg} \mathrm{L}^{-1} \mathrm{CCC}$ and $60.0 \mathrm{~g} \mathrm{~L}^{-1}$ sucrose, temperature $-6^{\circ} \mathrm{C}$. After 24 months of in vitro conservation in the genebank, explants of the lavender cultivar 'Sineva' maintained high viability (70-90\%) and reduced growth kinetics by two times compared to the control (Fig. 1). 

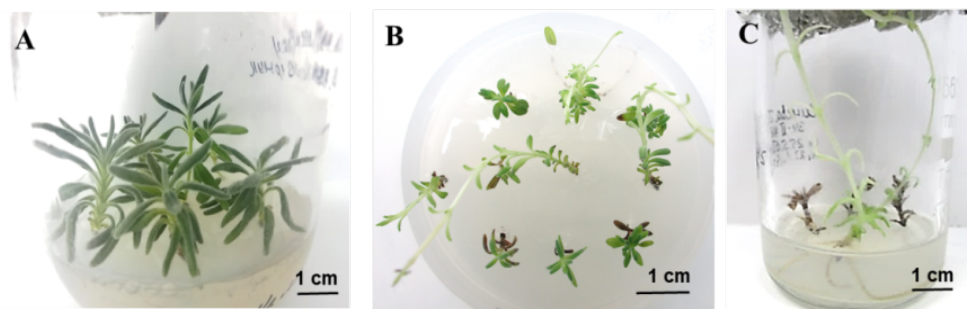

Fig. 1. Plant material of lavender cultivar 'Sineva': A - microshoots cultured in vitro during 6 months under standard conditions; B (microshoots) and C (spontaneous rooting of microshoots) - after 24 months conservation at in vitro genebank

Most explants demonstrated a slight elongation of the microshoots $(2.40-2.93 \mathrm{~cm})$, formation of leaves (6-9 ones/shoot) and roots (1-2 ones/shoot, length up to $3.20 \mathrm{~cm}$ ). Single plants had shoots up to $4.5 \mathrm{~cm}$ long with elongated internodes. At the same time, on the cross-sections of microshoots their round or oval shape, clear pith, dermal, vascular and parenchyma tissues were visible (Fig. 2). The dermal tissue of the shoots was represented by a single layer of closed epidermal cells with somewhat thickened outer walls. Some cells formed simple and glandular hairs. Beneath, there were cortex parenchyma cells (chlorenchyma), rarely collenchyma cells were found. The absence of clear mechanical tissues was noted. Endodermis consisted of 1-2 cell layers. Vascular system arranged as a ring, represented by xylem and phloem. The pith consisted of parenchyma cells with thin membranes.
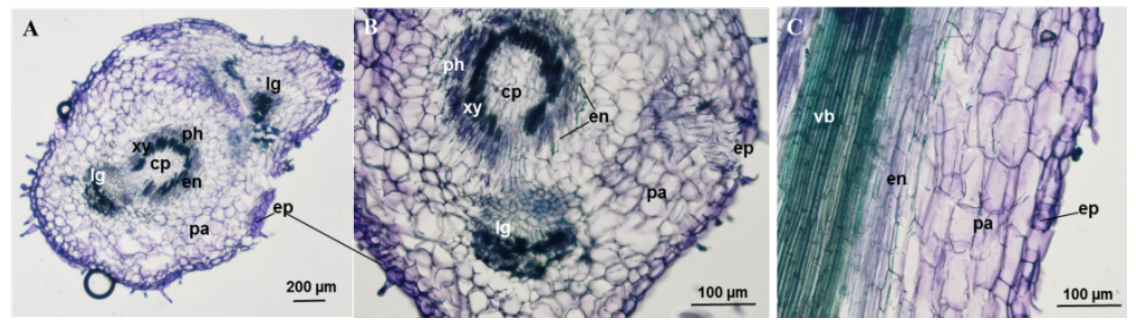

Fig. 2. Slides of in vitro deposited shoots of lavender cultivar 'Sineva': A, B - cross sections, C longitudinal sections; Ep - epidermis, pa - parenchyma, en - endoderma, $\mathrm{xy}$ - xylem, ph - phloem, lg - leaf gap, $\mathrm{cp}$ - central cylinder paranchyma, vb - vascular bundle

Compared to the standard culture conditions in vitro, it was noted a trend to decrease the number of collenchyma cells and ones of the central cylinder, as well as layers of the cortical parenchyma; no significant differences in the structure of the microshoots were found. It was revealed a significant decrease (by 1.7-2.5 times compared to the control) in the linear size of leaves in deposited lavender plants. The length of the leaf blade did not exceed $0.7 \mathrm{~cm}$. The shape of the leaves varied from a typical narrow-linear to oblong oval, sometimes - linear (Fig. 1).

Both in standard in vitro culture conditions and under prolonged deposition, a dorsiventral type of leaf structure was noted. On the cross sections of the leaf blades, one can noted epidermis with trichomes, vascular bundles, chlorenchyma, phloem, xylem and mechanical tissue (Fig. 3). Under micropropagation and deposition in vitro mesophyll included 3-4 cell layers. Under standard conditions, chlorenchyma was differentiated into palisade and spongy tissue (Fig. 3A), and under prolonged conservation in the genebank, it was represented by rounded mesophyll cells without clearly expressed differentiation (Fig. 3B). It should be noted that under the deposition, the leaf blade thickness slightly increased (from $129 \mu \mathrm{m}$ in the control to $164 \mu \mathrm{m}$ in the experiment), in some leaves an increase in the 
mesophyll intercellular space was noted. Histological analysis demonstrated a decrease in the number of chloroplasts in mesophyll cells and their wall location. These features correspond with a slowdown in the growth processes in the studied explants due to the inhibition of active photoassimilation processes [14]. The number of small vascular bundles in leaves decreased from 6-8 to 2-4, they were rarely accompanied by strands of mechanical tissue. Dermal tissues were thinner than in the case of cultivation under standard in vitro conditions. The cuticle was not clear: its average thickness decreased from $3.2 \mu \mathrm{m}$ to 0.8 $\mu \mathrm{m}$. Trichomes were less branched, often consisted of 3-4 cells. Under in vitro conditions, lavender leaves were of amphistomatic type and stomatal apparatuses were of the anomocytic type. The number of stomata was lower in plants conserved in the genebank in vitro: $49-56$ stomata/mm2 were on the abaxial side, while under standard in vitro conditions, they were 142-164 stomata/mm2

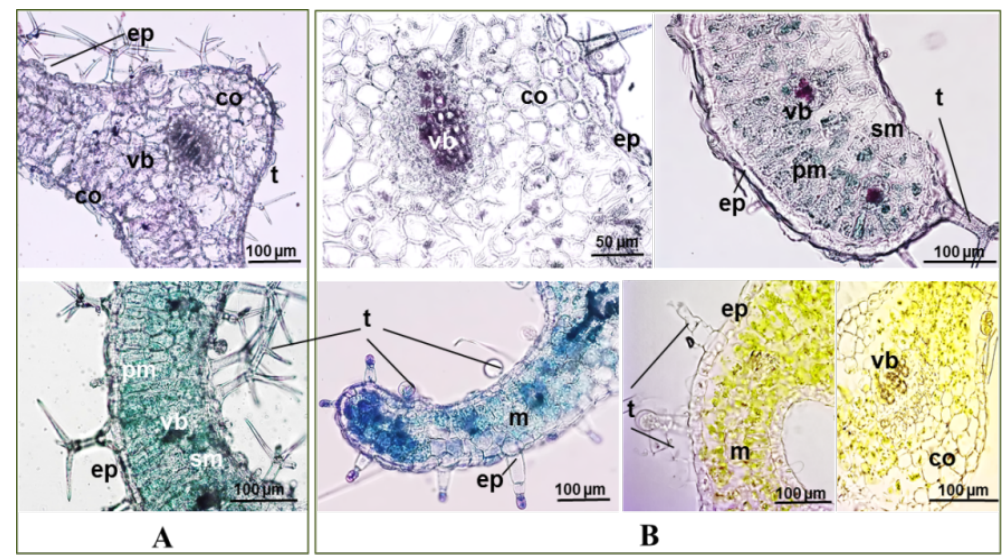

Fig. 3. Slides of the leaf blades from the microshoots of lavender cultivar 'Sineva': A - after in vitro culture during 6 months under standard conditions; $\mathrm{B}$ - after conservation under in vitro genebank conditions; ep - epidermis, vb - vascular bundle, $\mathrm{t}$ - trichoma, $\mathrm{m}$ - mesophile, $\mathrm{sp}$ - spongymesophile, $\mathrm{pm}$ - palisade mesophile, co - collenchyma.

\section{Conclusions}

Thus, on the example of the lavender cultivar 'Sineva', it was demonstrated that effective conservation of lavender plants in the genebank in vitro is possible with the correct choice of plant growth inhibitors in the culture medium and storage temperature, as well as taking into account both morphometric characteristics of the stored plants and their functional state. Changes in morphology and anatomy of leaves make it possible to suppose the high plasticity of the studied genotype to various in vitro culture conditions, and the presence of clear chlorenchyma indicates the ability to restart assimilation after prolonged deposition.

In vitro plant conservation study was funded by SA № 0829-2019-0038 of the FSFIS "NBG-NSC" and microscopy investigation of leaves in stored plants was supported by a research grant № 19-7600023 of the Russian Science Foundation. All work was done at the Unique Scientific Installation "Scientific Center of Plant Biotechnology, Genomics and Conservation" of the FSFIS "NBG-NSC".

\section{References}

1. M.F. Fay, Biodiversity and Conservation 3, 176 (1994)

2. O. Molkanova, D. Egorova, I. Mitrofanova, In Vitro Cellular \& Developmental Biology - 
Plant, 58, 46 (2018)

3. B. Borkowska, Acta Hortic., 530, 333 (2000)

4. I. Iliev, P. Kitin, Plant growth regulation, 63, 115 (2011

5. V.A. Brailko, O.V. Mitrofanova, N.V. Smykova, I.V. Mitrofanova, Acta Hortic., 1201, 607 (2018)

6. R.N. Trigiano, D.J. Gray Plant Development and Biotechnology (CRC Press, 2004)

7. O.V. Mitrofanova, N.P. Lesnikova-Sedoshenko, N.N. Ivanova, N.V. Smykova, I.V. Mitrofanova, Acta Hortic., 1285, 139 (2020)

8. F. Engelmann, Resources, 2, 73 (2013)

9. I.V. Mitrofanova, Fundamentals of in vitro genebank creation of species, cultivars and forms in ornamental, aromatic and fruit crops (Arial, Simferopol, 2018)

10. I.V. Mitrofanova, Modeling of controlled conditions necessary for adaptation and longterm storage of plant material of ornamental, aromatic and fruit crops in the in vitro genebank: methodological recommendations (Arial, Simferopol, 2018)

11. T. Murashige, F. Skoog. Physiol. Plant., 15, 473 (1962)

12. I.V. Mitrofanova, S.N. Chirkov, N.P. Lesnikova-Sedoshenko, S.V. Chelombit, A.V. Zakubanskiy, V.D. Rabotyagov, O.V. Mitrofanova, Acta Hortic., 1187, 37 (2017)

13. L.I. Lotova, Morphology and anatomy of higher plants (Editorial URSS, Moscow, 2001)

14. L.E. Lars, M. Tara, S. Sutinen, A.K. Clarke. Plant Physiology, 136, 4114 (2004) 